\title{
ON MINIMUM AND MAXIMUM OF FUNCTIONS OF SMALL BAIRE CLASSES
}

\author{
Atok Zulijanto
}

\begin{abstract}
A real-valued function on a Polish space $X$ is said to of Baire class one (or simply, a Baire-1 function) if it is the pointwise limit of a sequence of continuous functions. Let $\mathcal{B}_{1}(X)$ be the set of all real-valued Baire- 1 functions on $X$. Kechris and Louveau defined the set of functions of small Baire class $\xi$ for each countable ordinal $\xi$ as $\mathcal{B}_{1}^{\xi}(X)=\left\{f \in \mathcal{B}_{1}(X): \beta(f) \leq \omega^{\xi}\right\}$, where $\beta(f)$ denotes the oscillation index of $f$. In this paper we prove that the minimum and maximum of two functions of small Baire class $\xi$ are also functions of small Baire class $\xi$. This extends a result of Chaatit, Mascioni, and Rosenthal [1] who obtained the result for $\xi=1$.
\end{abstract}

\section{INTRODUCTION}

Let $X$ be a metrizable space. A function $f: X \rightarrow \mathbf{R}$ is said to be of Baire class one (or simply, a Baire-1 function) if it is the pointwise limit of a sequence of continuous functions on $X$. The Baire Characterization Theorem states that if $X$ is a Polish space, that is, a separable completely metrizable space, then $f: X \rightarrow \mathbf{R}$ is of Baire class one if and only if $\left.f\right|_{F}$ has a point of continuity for every nonempty closed subset $F$ of $X$. This leads naturally to the oscillation index for Baire-1 functions. This ordinal index was used by Kechris and Louvaeu [2] to give a finer gradation of Baire-1 functions into small Baire classes. Let $\mathcal{B}_{1}(X)$ be the set of all Baire-1 functions on $X$. For every ordinal $\xi<\omega_{1}$, the set of functions of small Baire class $\xi$ is defined as

$$
\mathcal{B}_{1}^{\xi}(X)=\left\{f \in \mathcal{B}_{1}(X): \beta(f) \leq \omega^{\xi}\right\}
$$

This study was continued by various authors. (See, e.g., [3],[4], and [5]).

Received 17-10-2009, Accepted 15-01-2010.

2000 Mathematics Subject Classification: Primary 26A21; Secondary 03E15, 54C30

Key words and Phrases: Baire class one functions, oscillation index 
In this paper, we prove that if $f$ and $g$ belong to a small Baire class $\xi$ for some $\xi<\omega_{1}$, then the minimum and maximum of $f$ and $g$ also belong to that class. This extends a result of Chaatit, Mascioni and Rosenthal [1] who obtained the result for $\xi=1$.

We begin by recalling the definition of oscillation index $\beta$. The oscillation index $\beta$ is associated with a family of derivations. Let $X$ be a metrizable space and $\mathcal{C}$ denote the collection of all closed subsets of $X$. A derivation is a map $\mathcal{D}: \mathcal{C} \rightarrow \mathcal{C}$ such that $\mathcal{D}(H) \subseteq H$ for all $H \in \mathcal{C}$. Let $\varepsilon>0$ and a function $f: X \rightarrow \mathbf{R}$ be given. For any closed subset $H$ of $X$ set $\mathcal{D}^{0}(f, \varepsilon, H)=H$ and $\mathcal{D}^{1}(f, \varepsilon, H)$ be the set of all $x \in H$ such that for every open set $U$ containing $x$ there are two points $x_{1}$ and $x_{2}$ in $U \cap H$ with $\left|f\left(x_{1}\right)-f\left(x_{2}\right)\right| \geq \varepsilon$. For $\alpha<\omega_{1}$, let

$$
\mathcal{D}^{\alpha+1}(f, \varepsilon, H)=\mathcal{D}^{1}\left(f, \varepsilon, \mathcal{D}^{\alpha}(f, \varepsilon, H)\right) .
$$

If $\alpha$ is a countable limit ordinal,

$$
\mathcal{D}^{\alpha}(f, \varepsilon, H)=\bigcap_{\alpha^{\prime}<\alpha} \mathcal{D}^{\alpha^{\prime}}(f, \varepsilon, H)
$$

The $\varepsilon$-oscillation index of $f$ on $H$ is defined by

$$
\beta_{H}(f, \varepsilon)=\left\{\begin{array}{l}
\text { the smallest ordinal } \alpha<\omega_{1} \text { such that } \mathcal{D}^{\alpha}(f, \varepsilon, H)=\emptyset \\
\text { if such an } \alpha \text { exists } \\
\omega_{1}, \text { otherwise. }
\end{array}\right.
$$

The oscillation index of $f$ on the set $H$ is defined by

$$
\beta_{H}(f)=\sup \left\{\beta_{H}(f, \varepsilon): \varepsilon>0\right\} .
$$

We shall write $\beta(f, \varepsilon)$ and $\beta(f)$ for $\beta_{X}(f, \varepsilon)$ and $\beta_{X}(f)$ respectively.

\section{MAIN RESULTS}

Throughout, let $X$ be a Polish space. For $f, g: X \rightarrow \mathbf{R}$, we denote their minimum and their maximum by $f \wedge g$ and $f \vee g$ respectively. A result in [1] is that if the oscillation indices of $f$ and $g$ are finite then the oscillation indices of $f \wedge g$ and $f \vee g$ are also finite. We extend this result into the classes of small Baire functions. We get the following result.

Theorem 2.1. Let $f, g: X \rightarrow \mathbf{R}$. If $\beta(f) \leq \omega^{\xi}$ and $\beta(g) \leq \omega^{\xi}$ for some $\xi<\omega_{1}$, then $\beta(f \wedge g) \leq \omega^{\xi}$ and $\beta(f \vee g) \leq \omega^{\xi}$.

Theorem 2.1 is proved by the method used in [2]. Following [5], we define a derivation $\mathcal{G}$ which closely related to $\mathcal{D}$. Given a real-valued function $f$ on $X$, 
$\varepsilon>0$, and a closed subset $H$ of $X$. Define $G(f, \varepsilon, H)$ to be the set of all $x \in H$ such that for any open neighborhood $U$ of $x$, there exists $x^{\prime} \in H \cap U$ such that $\left|f(x)-f\left(x^{\prime}\right)\right| \geq \varepsilon$. Let

$$
\mathcal{G}^{1}(f, \varepsilon, H)=\overline{G(f, \varepsilon, H)}
$$

where the closure is taken in $X$. If $\alpha<\omega_{1}$, let

$$
\mathcal{G}^{\alpha+1}(f, \varepsilon, H)=\mathcal{G}^{1}\left(f, \varepsilon, \mathcal{G}^{\alpha}(f, \varepsilon, H)\right) .
$$

If $\alpha<\omega_{1}$ is a limit ordinal, let

$$
\mathcal{G}^{\alpha}(f, \varepsilon, H)=\bigcap_{\alpha^{\prime}<\alpha} \mathcal{G}^{\alpha^{\prime}}(f, \varepsilon, H) .
$$

The relationship between derivations $\mathcal{D}$ and $\mathcal{G}$ is given in the following lemma that can be seen in [5, Lemma 4].

Lemma 2.2. If $f$ be real-valued function on $X, \varepsilon>0$ and $H$ is a closed subset of $X$, then

$$
\mathcal{D}^{\alpha}(f, 2 \varepsilon, H) \subseteq \mathcal{G}^{\alpha}(f, \varepsilon, H) \subseteq \mathcal{D}^{\alpha}(f, \varepsilon, H),
$$

for all $\alpha<\omega_{1}$.

Before we prove the main result, we show the following results first.

Lemma 2.3. If $f_{1}$ and $f_{2}$ are real-valued functions on $X, \varepsilon>0, H$ is a closed subset of $X$ and $f=f_{1} \wedge f_{2}$ then

$$
\mathcal{G}^{1}(f, \varepsilon, H) \subseteq \mathcal{G}^{1}\left(f_{1}, \varepsilon, H\right) \cup \mathcal{G}^{1}\left(f_{2}, \varepsilon, H\right) .
$$

Proof. Let $x \in G(f, \varepsilon, H)$. If $U$ is an open neighborhood of $x$ in $X$ then there exists $x^{\prime} \in U \cap H$ such that $\left|f(x)-f\left(x^{\prime}\right)\right| \geq \varepsilon$. If $\left|f(x)-f\left(x^{\prime}\right)\right|=f(x)-f\left(x^{\prime}\right)$, then

$$
\begin{aligned}
\left|f(x)-f\left(x^{\prime}\right)\right| & =f_{i}(x)-f_{j}\left(x^{\prime}\right), \quad i, j \in\{1,2\} \\
& \leq f_{j}(x)-f_{j}\left(x^{\prime}\right), \quad j \in\{1,2\} \\
& =\left|f_{j}(x)-f_{j}\left(x^{\prime}\right)\right|, \quad j \in\{1,2\} .
\end{aligned}
$$

Therefore $\left|f_{j}(x)-f_{j}\left(x^{\prime}\right)\right| \geq \varepsilon, \quad j \in\{1,2\}$. This shows $x \in G\left(f_{1}, \varepsilon, H\right) \cup G\left(f_{2}, \varepsilon, H\right)$. Similarly, whenever $\left|f(x)-f\left(x^{\prime}\right)\right|=f\left(x^{\prime}\right)-f(x)$.

It follows that

$$
\mathcal{G}^{1}(f, \varepsilon, H)=\mathcal{G}^{1}\left(f_{1}, \varepsilon, H\right) \cup \mathcal{G}^{1}\left(f_{2}, \varepsilon, H\right) .
$$

Similarly, we obtain the following lemma. 
Lemma 2.4. If $f_{1}$ and $f_{2}$ are real-valued functions on $X, \varepsilon>0, H$ is a closed subset of $X$ and $f=f_{1} \vee f_{2}$ then

$$
\mathcal{G}^{1}(f, \varepsilon, H) \subseteq \mathcal{G}^{1}\left(f_{1}, \varepsilon, H\right) \cup \mathcal{G}^{1}\left(f_{2}, \varepsilon, H\right) .
$$

Now, we are ready to prove the main result.

Proof of Theorem 2.1. We prove for the minimum of $f$ and $g$, for the $f \vee g$ we can prove in the similar way, by using Lemma 2.4 instead of Lemma 2.3. Let $\varepsilon>0$. First, we prove that

$$
\mathcal{G}^{\omega^{\xi}}(f \wedge g, \varepsilon, H) \subseteq \mathcal{G}^{\omega^{\xi}}(f, \varepsilon, H) \cup \mathcal{G}^{\omega^{\xi}}(g, \varepsilon, H) .
$$

for all closed subset $H$ of $X$ and $\xi<\omega_{1}$.

We prove (1) by transfinite induction on $\xi$. For $\xi=0$, i.e., $\omega^{\xi}=1$, this just Lemma 2.3. Since $\left(\mathcal{G}^{\alpha}(f, \varepsilon, H)\right)_{\alpha}$ and $\left(\mathcal{G}^{\alpha}(g, \varepsilon, H)\right)_{\alpha}$ are non-increasing, then (1) is immediate for a limit ordinal $\xi<\omega_{1}$.

Suppose that (1) is true for some ordinal $\xi<\omega_{1}$, we have to prove that (1) is also true for $\xi+1$. For this, we need to prove that

$$
\mathcal{G}^{\omega^{\xi} \cdot 2 n}(f \wedge g, \varepsilon, H) \subseteq \mathcal{G}^{\omega^{\xi} \cdot n}(f, \varepsilon, H) \cup \mathcal{G}^{\omega^{\xi} \cdot n}(g, \varepsilon, H)
$$

for all $n \in \mathbf{N}$.

For this, let for $s \in 2^{k}=\left\{\left(\epsilon_{1}, \epsilon_{2}, \ldots, \epsilon_{k}\right): \epsilon_{i}=0\right.$ or 1$\}, k \in \mathbf{N}$, we define $H_{s}$ as follows

$$
\begin{aligned}
& H_{0}=\mathcal{G}^{\omega^{\xi}}(f, \varepsilon, H), \\
& H_{1}=\mathcal{G}^{\omega^{\xi}}(g, \varepsilon, H),
\end{aligned}
$$

and

$$
\begin{aligned}
& H_{s^{\wedge} 0}=\mathcal{G}^{\omega^{\xi}}\left(f, \varepsilon, H_{s}\right), \\
& H_{s^{\wedge} 1}=\mathcal{G}^{\omega^{\xi}}\left(g, \varepsilon, H_{s}\right) .
\end{aligned}
$$

In order to prove (2), we need to show that

$$
\mathcal{G}^{\omega^{\xi} \cdot k}(f \wedge g, \varepsilon, H) \subseteq \bigcup_{s \in 2^{k}} H_{s}
$$

for all $k \in \mathbf{N}$. By the assumption induction, statement (3) is true for $k=1$. 
Suppose that (3) is true fo some $k \in \mathbf{N}$. We obtain

$$
\begin{aligned}
\mathcal{G}^{\omega^{\xi} \cdot(k+1)}(f \wedge g, \varepsilon, H) & =\mathcal{G}^{\omega^{\xi} \cdot k+\omega^{\xi}}(f \wedge g, \varepsilon, H) \\
& =\mathcal{G}^{\omega^{\xi}}\left(f \wedge g, \varepsilon, \mathcal{G}^{\omega^{\xi} \cdot k}(f \wedge g, \varepsilon, H)\right) \\
& \subseteq \mathcal{G}^{\omega^{\xi}}\left(f \wedge g, \varepsilon, \bigcup_{s \in 2^{k}} H_{s}\right) \\
& \subseteq \bigcup_{s \in 2^{k}} \mathcal{G}^{\omega^{\xi}}\left(f \wedge g, \varepsilon, H_{s}\right) \text { by }[5, \text { Lemma 4] } \\
& \subseteq \bigcup_{s \in 2^{k}}\left(\mathcal{G}^{\omega^{\xi}}\left(f, \varepsilon H_{s}\right) \cup \mathcal{G}^{\omega^{\xi}}\left(g, \varepsilon, H_{s}\right)\right. \\
& =\left(\bigcup_{s \in 2^{k}} \mathcal{G}^{\omega^{\xi}}\left(f, \varepsilon, H_{s}\right) \cup\left(\bigcup_{s \in 2^{k}} \mathcal{G}^{\omega^{\xi}}\left(g, \varepsilon, H_{s}\right)\right)\right. \\
& =\left(\bigcup_{s \in 2^{k}} H_{s^{\wedge} 0}\right) \cup\left(\bigcup_{s \in 2^{k}} H_{s^{\wedge} 1}\right) \\
& =\bigcup_{s \in 2^{k+1}} H_{s} .
\end{aligned}
$$

By (3), for all $n \in \mathbf{N}$, we have

$$
\begin{aligned}
\mathcal{G}^{\omega^{\xi} \cdot 2 n}(f \wedge g, \varepsilon, H) \subseteq & \bigcup_{s \in 2^{2 n}} H_{s} \\
\subseteq & \bigcup\left\{H_{s}: s \in 2^{2 n} \text { dan card }(\{k: s(k)=0\}) \geq n\right\} \\
& \cup \bigcup\left\{H_{s}: s \in 2^{2 n} \text { dan } \operatorname{card}(\{k: s(k)=1\}) \geq n\right\} .
\end{aligned}
$$

If $s$ takes at least $n$ values 0 , then $H_{s} \subseteq \mathcal{G}^{\omega^{\xi} \cdot n}(f, \varepsilon, H)$. Similarly, if $s$ takes at least $n$ values 1 , then $H_{s} \subseteq \mathcal{G}^{\omega^{\xi} \cdot n}(g, \varepsilon, H)$. Therefore, the proof of (2) is finished.

Since $\left(\mathcal{G}^{\alpha}(f, \varepsilon, H)\right)_{\alpha}$ and $\left(\mathcal{G}^{\alpha}(g, \varepsilon, H)\right)_{\alpha}$ are non-increasing, then by taking the intersection over $n$ in (2) gives

$$
\mathcal{G}^{\omega^{\xi+1}}(f \wedge g, \varepsilon, H) \subseteq \mathcal{G}^{\omega^{\xi+1}}(f, \varepsilon, H) \cup \mathcal{G}^{\omega^{\xi+1}}(g, \varepsilon, H)
$$

Using (1) and Lemma 2.1, since $\beta(f) \leq \omega^{\xi}$ and $\beta(g) \leq \omega^{\xi}$, then

$$
\mathcal{G}^{\omega^{\xi}}(f, \varepsilon, H)=\emptyset \operatorname{dan} \mathcal{G}^{\omega^{\xi}}(g, \varepsilon, H)=\emptyset .
$$

Therefore,

$$
\mathcal{D}^{\omega^{\xi}}(f \wedge g, 2 \varepsilon, H) \subseteq \mathcal{G}^{\omega^{\xi}}(f \wedge g, \varepsilon, H)=\emptyset .
$$

It follows that $\beta(f \wedge g) \leq \omega^{\xi}$. 


\section{REFERENCES}

1. F. Chantit, V. Mascioni and H.P. Rosenthal, "On functions of finite Baire index", J. Funct. Anal. 142 (1996), 277-295.

2. A.S. Kechris And A. Louveau, "A classification of Baire class 1 functions", Trans. Amer. Math. Soc. 318 (1990), 209-326.

3. P. Kiriakouli, "A classification of Baire-1 functions", Trans. Amer. Math. Soc. 351 (1999), 4599-4609.

4. D.H.Leung AND W.-K.TANG, "Functions of Baire class one", Fund. Math. 179 (2003), 225-247.

5. D.H.Leung And W.-K.TAng, "extension of functions with small oscillation", Fund. Math. 192 (2006), 183-193.

Atok Zulijanto: Department of Mathematics, Faculty of Mathematics and Natural Sciences, Gadjah Mada University, Yogyakarta, Indonesia.

E-mail: atokzulijanto@yahoo.com 